\title{
Binary Artificial Bee Colony Algorithm to Solve Single Objective Resource Allocation Problem
}

\author{
Zuleyha Yilmaz and Fatih Basciftci
}

\begin{abstract}
Resource allocation problem (RAP) is an optimum distributing period of limited existing resources. In this problem, there can be two possible cases: the resource is allocated or not. If so, the problem is in 0-1 integer problem class of which decision variables are binary values, and binary optimization algorithms are used to find optimum solutions for these problems. In the literature, various meta-heuristic optimization methods have been proposed for solving continuous or discrete RAP. In this paper, Artificial Bee Colony (ABC) algorithm simulated foraging behavior of real honey bees is studied to optimize RAP. The algorithm is firstly developed to solve numeric optimization problems and cannot be applied to binary problems directly. It requires a modification. In this study, the ABC algorithm has become applicable to solve the RAP and is called Binary Artificial Bee Colony (BABC) algorithm. The BABC algorithm is compared with the binary particle swarm optimization algorithm (BPSO) which is applied to the problem previously. The experimental results showed that the $\mathrm{BABC}$ algorithm was superior and can be alternative optimization method for solving RAP.
\end{abstract}

Index Terms-Artificial bee colony algorithm, binary optimization, meta-heuristic algorithm, resource allocation problem.

\section{INTRODUCTION}

Resource allocation problem (RAP) is an optimum distribution planning of restricted available resource to activities in order to minimize the cost or maximize utilization [1]. The resources can be human resource, assets, capital, machine or any resource to realize intended objective. There are many RAP types in the literature, including software testing [2], parallelizable tasks scheduling [3], bandwidth allocation [4], internet protocol address allocation [5].

In this paper, job shop scheduling problem (JSP) which is a kind of RAP, where operations (jobs) are processed on available machines, is selected. In the problem, finding optimal solution is difficult when there are many operations and machines. Hence JSP is a well-known NP-hard problem [6]. Since allocation of many jobs to machines is complicated, many approaches and methods are performed to solve the JSP. Firstly, branch and bound algorithms have been introduced in [7]-[9]. Then, heuristic algorithms have been developed for solving the JSP. Some of them are Simulating Annealing [10], Taboo Search [11], Genetic Algorithm [12], PSO Algorithm [13]. In this study, the same problem and

Manuscript received February 12, 2018; revised March 25, 2018.

Z. Yilmaz is with the Department of Computer Engineering, Selcuk University, Konya, Turkey (e-mail: zuleyhayilmaz@selcuk.edu.tr).

F. Basciftci is with the Department of Computer Engineering, Selcuk University, Konya, Turkey (e-mail: basciftci@selcuk.edu.tr). mathematical structure as [14] is carried out. Accordingly, human resources are allocated to jobs by applying proposed algorithm. The problem incorporates $\mathrm{n}$ jobs and $\mathrm{m}$ workers. The objective is to find optimum solutions to minimize completion time. In problem, there is only one worker which the job is processed by at a time. Moreover, a worker can be processed more than one job.

In the problem, there are two discrete case: the job is assigned to a worker or not. Namely, the problem can be identified 0-1 integer values and is called binary optimization problem. If the job is assigned to the worker, decision variable is set to 1 . On the other hand, 0 indicates that job is not assigned the worker.

This study focuses on Artificial Bee Colony algorithm (ABC) which is swarm based and simulates foraging behavior of real honey bees. As the problem is a binary optimization problem, selected algorithm should be implemented into binary space. Therefore, the algorithm is become applicable to solve the problem. The modified algorithm is utilized to the problem in order to find minimum completion time in the study.

In this paper, firstly described the basic $\mathrm{ABC}$ and binary version of $\mathrm{ABC}$; then introduced the problem and mathematical formulation; then, represented solving the problem using the Binary Artificial Bee Colony (BABC). Finally, reported experimental results and conclusions.

\section{BinARy ARtificial BeE Colony Algorithm (BABC)}

\section{A. The Basic Concept of ABC}

$\mathrm{ABC}$ is a numerical stochastic optimization algorithm inspired from foraging behavior of real bee swarm. The ABC, is firstly for real-parameter optimization, has been developed by Karaboga in 2005 [15]. The algorithm consists of three group artificial bees. Their names and tasks are as follows: First group is employed bees. Mission of the bees is exploiting food sources and sharing knowledge about the source with the others which are awaiting in the beehive. The knowledge is used by onlooker bees which are second group of the artificial bees. Choosing a food source by the bees depends on the information. The more nectar amount, the higher possibility of being selected. The last bee group is scout bees. In the ABC algorithm, if a food source is exhausted, the employed bee of the food source transforms into a scout bee. New mission is trying to find a new source randomly for the bee.

In basic concept of the algorithm, employed and onlooker bees constitute the whole colony. If $\mathrm{N}$ denotes the colony size, $\mathrm{N} / 2$ denotes respectively employed bee and onlooker bee 
numbers. According to the algorithm, it is assumed that an employed bee is responsible for only one food source. Locations of the sources refer to the probable solutions of problems. Nectar amounts of sources represent quality of solutions. In the algorithm, food source which has the most nectar amount is the optimum solution.

Initialization phase: swarm size, termination criteria and limit (exhaustion counter) are determined in the initial phase. The initial population is generated by randomly as follows:

$$
x_{i j}=x_{j}^{\min }+\operatorname{rand}(0,1)\left(x_{j}^{\max }-x_{j}^{\min }\right)
$$

$X_{i j}$ is decision variable. $i$ denotes the number of food source (SN, the half of swarm size) and $j$ denotes the dimension of problem (D). $x_{j}^{\min }$ and $x_{j}^{\max }$ denote lower bound and upper bound of each dimension.

Employed bee phase: Calculation nectar quantity of neighbour source occurs in this phase. It is defined by (2):

$$
v_{i j}=x_{i j}+\phi_{i j}\left(x_{i j}-x_{k j}\right)
$$

$X$ is the existing food source, $v$ is the new food source, $\phi_{i j}$ is randomly determined in the interval between $[-1,1], k$ and $j$ are random integers in the range respectively $[1, \mathrm{SN}]$ and $[1$, D].

After producing $v$ value, the fitness value is determined for minimization problem as (3). The found value belongs to the new food source.

$$
\text { fitness }_{i}= \begin{cases}1 /\left(1+f_{i}\right), & f_{i} \geq 0 \\ 1+a b s\left(f_{i}\right), & f_{i} \prec 0\end{cases}
$$

$F_{i}$ is cost value, for maximization problem the cost value is directly used, for minimization problem fitness value is used.

In $\mathrm{ABC}$, if there is more nectar amount than existing source in the new food source, the new is stored and the other is removed. Vice versa, existing food source is still being stored, however the exhaustion counter of the existing source is increased.

Onlooker bee phase: this phase is occurred by onlooker bees. The choice of a food source depends on the its quality. This probability is calculated by roulette wheel selection method as (4):

$$
p_{i}=f i t_{i} / \sum_{j=1}^{S N} f i t_{j}
$$

Owing to this method, the probability of being selected food source which has better fitness value becomes higher.

Scout bee phase: End of every cycle, the exhaustion counter of every food source is controlled. If there is a source reached limit value, employed bee which exploits the source is anymore called as a scout bee. This means the nectar was consumed in the source, and it is replaced with new random one explored by the scout bee.

\section{B. Binary Artificial Bee Colony Algorithm}

In the problem, decision variables consist of 0-1 binary values. If the variable is 0 , it means the job is not assigned the worker. On the other hand, if the variable is 1 , it means the job is assigned the worker. Due to the fact that the problem is a binary optimization problem, the algorithm should be binary optimization algorithm. This section explains BABC for binary optimization. The ABC is firstly proposed for numeric problems, and some modifications are required in the algorithm. Initial population and also definition of producing new food source should swap with binary values in BABC.

First of all, initial population is generated by (5) instead of

$$
x_{i j}=\left\{\begin{array}{l}
0 \text { if } \operatorname{rand}(0,1) \leq 0.5 \\
1 \text { if } \operatorname{rand}(0,1) \succ 0.5
\end{array}\right.
$$

where $i=1, \ldots, S N ; j=1, \ldots, D$.

In this study, Sigmoid transition function is utilized for converted to binary space. Since food sources defines as 0-1 integer values, also new food sources to be found should be defined as $0-1$ values. In the BABC, new food source definition is defined as (6) through Sigmoid function instead of (2);

$$
\begin{aligned}
& \text { if rand } \prec \operatorname{sigm}\left(v_{i j}\right) \text { then } x_{i j}=1 \\
& \text { else } x_{i j}=0
\end{aligned}
$$

where the $\operatorname{sigm}\left(v_{i j}\right)$ is Sigmoid transformation function $\left.\operatorname{sigm}\left(v_{i j}\right)=1 /\left(1+\exp \left(-v_{i j}\right)\right)\right)$ and rand is set to in the range $[0,1]$.

Expect the changes on (1) and (2), other formulas remain unchanged to preserve originality of the algorithm in this study. In addition, these modifications implement all bee phases in proposed BABC.

\section{A KIND OF RAP: JSP}

In this study, in order to make an equitable comparison, the same mathematical model as [14] is used for human RAP. $M=\{1, \ldots, m\}$ indicates the human set and $N=\{1, \ldots, n\}$ indicates the job set. In the problem, there are $m$ workers and $n$ jobs.

The problem consists of two parts: deterministic and stochastic part. In deterministic model, processing times, setup times and skill coefficients are deterministic and independent. The objective is to find optimal solution to minimize completion time. In stochastic model, processing times and setup times are modelled by random values. The optimal minimum expectation value is the objective as deterministic model [14].

In deterministic model, mathematical formulation is as follows;

$$
\begin{gathered}
\text { Minimize } C_{\max }=\max \left(\begin{array}{l}
\left.\sum_{j=1}^{n}\left(k_{1} t_{j}+S T_{j}\right) X_{1 j}, \ldots, \sum_{j=1}^{n}\left(k_{i} t_{j}+S T_{j}\right) X_{i j}, \ldots,\right) \\
\sum_{j=1}^{n}\left(k_{m} t_{j}+S T_{j}\right) X_{m j},
\end{array}\right) \\
\sum_{i=1}^{m} X_{i j}=1, j=1,2, \ldots, n
\end{gathered}
$$

The workers are assigned to the jobs. The total time spent for the jobs by a worker is called completion time $C_{i}$. In the 
problem, the maximum time is required among them and is called $C_{\max }$. So as to minimize the $C_{\max }$ equation (7) is used, where $\mathrm{k}$ is the skill coefficient. ST is setup time and shortest processing time of a job is indicated by $t$. Constraint (8) utilizes that a job can be assigned to only one worker. Constraint (9) utilizes that number of jobs processed by a worker is between zero and number of jobs. Decision variable is $X_{i j}$ (10). If $X_{i j}$ is 1 , it means that job $j$ is assigned to worker $i$, or vice versa. These equations belong to deterministic model of the problem.

In stochastic model, the mathematical model is as follows;

$$
\begin{gathered}
C_{i}=\sum_{j=1}^{n}\left(T_{i j}+S T_{j}\right) X_{i j} \\
E\left(C_{i}\right)=\sum_{j=1}^{n}\left(T_{i j}+S T_{j}\right) X_{i j} \\
E\left(C_{i}\right)=\sum_{j=1}^{n}\left(1 / \mu_{i j}+1 / \phi_{i j}\right) X_{i j} \\
\text { Minimize } E\left(C_{\max }\right)=\max \left\{E\left(C_{1}\right), E\left(C_{2}\right),\right. \\
\left.\ldots, E\left(C_{m}\right)\right\}
\end{gathered}
$$

where $S T$ is setup time, $T$ is processing time of the jobs. Both ST and $T$ are randomly determined. $\mu$ and $\varphi$ are the distribution ratio for the processing time of the job. Equation (13) defines that the expected value of $C_{i}$ called $E\left(C_{i}\right)$ will be optimized to find minimum solution. In stochastic model, the constraints are the same as deterministic model definitions.

\section{Resource Allocation Using BABC}

This study focuses on solving RAP using BABC optimization. According to this algorithm, initial job-worker matching is randomly generated in initial phase. Each food source demonstrates a job-worker matching. Then, fitness values of initial solutions are evaluated.

As in employed bee phase, nectar quantity of neighbor source is determined. It refers to suggestion a variation of a dimension of a job-worker assignment array. The modification means that a job is assigned to another worker. If this modification has better fitness value (minimum completion time) than the existing food source -new food source- is memorized. Namely, suggestion of changing is accepted.

After neighbour investigation is completed by employed bees in a cycle, onlooker phase begins. According to fitness values of the sources, a new source is chosen by onlooker bees and try to improve it as employed bees.

In the last phase, all food sources are controlled about the exhausted number. If there is a poor job-worker matching, and any bee cannot improve it at the end of a cycle, the exhausted value of the food source can reach the limit. In such a case, a new food source is determined for the abandoned source, namely a new job-worker matching for JSP.

These algorithm steps continue until reaching the maximum iteration. Flow chart of the job scheduling with BABC is illustrated in Fig. 1.

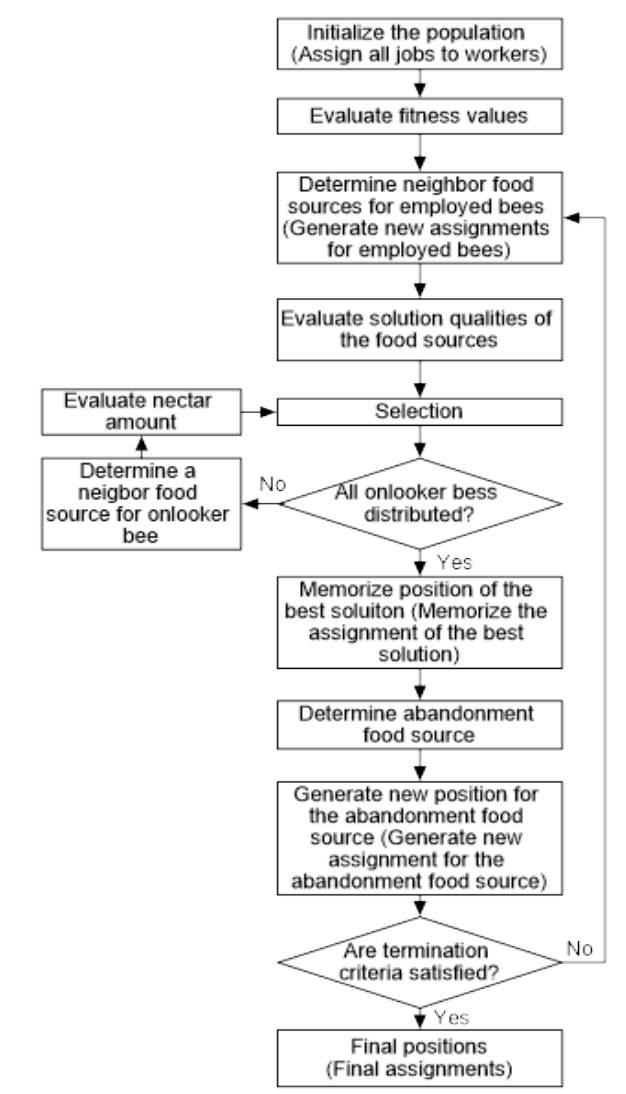

Fig. 1. Flow chart of resource allocation process with BABC.

\section{EXPERIMENTAL RESULTS}

In this section, the example simulation results of performance among BABC are presented for the resource allocation problem. In this study, to make an equitable comparison the same problem, human resource allocation, is optimized and all parameters are set as [14].

Data set of Table I belongs to case 1. Accordingly, there are 5 jobs and 3 workers [14]. By BABC, the problem is optimized in $0.025 \mathrm{~s}$ with optimum solution 14.2. In [14], the optimum solution was found in 0.03 by improved BPSO. We can say that the difference of the times of these algorithms can be negligible. In case 2, there has 100 jobs processed by 20 workers. The parameters are determined follows: shortest processing times of jobs are randomly set in $[6,10]$, setup time of jobs are randomly set in [2, 4] and last skill coefficients of workers are set randomly in the range [1, 3]. In ABC, swarm size is set as 400 , maximum iteration is taken as 500. The algorithm is run 10 times and all the results are showed in Table II. The maximum completion time clearly can be distinguished in the table; that is 87,66. In [14], maximum completion time has found as 108 . It expresses a decrease of $18,8 \%$. This reduction rate implying significant decrement for JSP shows the efficiency of the BABC.

In case 3, 20 jobs are processed by 5 workers; population size is 200 , maximum iteration is 200 . The parameters are set as $\mu$ is in the range $[0.2,1.0], \varphi$ is in the range $[0.1,0.5]$. The problem is optimized from 26.19 to 22.27 as from 50th iteration. In [14], the maximum completion time was optimized to 34.0. Our solution is showed efficiency of our proposed BABC. Fig. 2 illustrates optimization process of the maximum completion time of the JSP. 
TABLE I: THE ORIGINAL DATA OF THE CASE 1

\begin{tabular}{|c|c|c|c|c|c|c|c|c|c|c|}
\hline Worker & Job-1 & & Job-2 & & Job-3 & & Job-4 & & Job- & \\
\hline & $\mathrm{k}_{\mathrm{i}} \mathrm{t}_{1}$ & $\mathrm{ST}_{1}$ & $\mathrm{k}_{\mathrm{i}} \mathrm{t}_{2}$ & $\mathrm{ST}_{2}$ & $\mathrm{k}_{\mathrm{i}} \mathrm{t}_{3}$ & $\mathrm{ST}_{3}$ & $\mathrm{k}_{\mathrm{i}} \mathrm{t}_{4}$ & $\mathrm{ST}_{4}$ & $\mathrm{k}_{\mathrm{i}} \mathrm{t}_{5}$ & $\mathrm{ST}_{5}$ \\
\hline 1 & 5 & 2 & 3 & 1 & 8 & 3 & 6 & 2 & 4 & 1.5 \\
\hline 2 & 6 & 2 & 3.6 & 1 & 9.6 & 3 & 7.2 & 2 & 4.8 & 1.5 \\
\hline 3 & 7 & 2 & 4.2 & 1 & 11.2 & 3 & 8.4 & 2 & 5.6 & 1.5 \\
\hline
\end{tabular}

Skill coefficient (k), Number of worker (i), Shortest processing time (t), Setup time (ST)

TABLE II: REPRESENTING RESULTS IN CASE 2

\begin{tabular}{llllllllll}
\hline \hline Time & 1 & 2 & 3 & 4 & 5 & 6 & 7 & 8 & 9 \\
\hline $\mathrm{C}_{\max }(\mathrm{h})$ & 92.02 & 90.05 & 88.51 & 91.84 & 92.38 & 89.91 & 91.02 & 90.92 & 87.66 \\
\hline \hline
\end{tabular}

Maximum Completion Time $\left(\mathrm{C}_{\max }\right)$

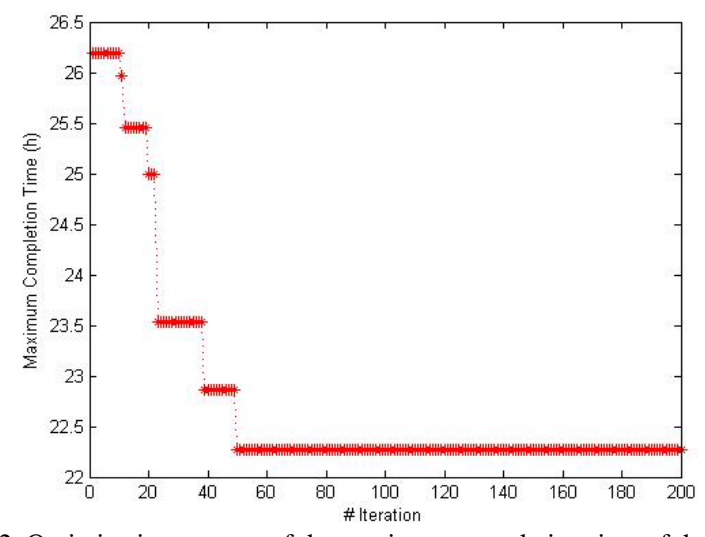

Fig. 2. Optimization process of the maximum completion time of the JSP according to the $\mathrm{BABC}$.

The comparison of all cases mentioned above is showed in Table III. For the JSP, which is a type of RAP and solved in this study, only BPSO algorithm is proposed. Because of that, comparison is made with only BPSO algorithm. As seen in the Table III, the proposed BABC algorithm obtained satisfactory results for the JSP.

In all cases, the BABC has been coded in Matlab, version R2016a by the author and all experiments have been performed on personal notebook on $3.1 \mathrm{GHz}$, Intel Core i7processor with 12GB.

TABLE III: THE RESULTS OBTAINED BY BABC AND BPSO FOR THE JSP

\begin{tabular}{llll}
\hline \hline \multirow{2}{*}{ Cases } & & The Proposed & BPSO \\
& & BABC & \\
\hline \multirow{3}{*}{ Case 1} & 5 & 5 \\
& Job & 3 & 3 \\
& Worker & 14.2 & 14.2 \\
& C $_{\text {max }}(\mathrm{h})$ & $\mathbf{0 . 0 2 5}$ & 0.03 \\
& Computing Time (s) & 100 & 100 \\
Case 2 & Job & 20 & 20 \\
& Worker & $\mathbf{8 7 . 6 6}$ & 108 \\
& $\mathrm{C}_{\max }(\mathrm{h})$ & 20 & 20 \\
& Job & 5 & 5 \\
Case 3 & Worker & $\mathbf{2 2 . 2 7}$ & 34.0 \\
& $\mathrm{C}_{\max }(\mathrm{h})$ &
\end{tabular}

Maximum Completion Time $\left(\mathrm{C}_{\max }\right)$

\section{CONCLUSION}

RAP is a kind of distribution among limited resources to minimize cost or maximize efficiency. There are lots of problem types of resource allocation such as software testing, IP address allocation, job shop scheduling.
In this paper, a kind of JSP [14] is optimized to find minimum completion time by BABC. The reasons for selecting the algorithm are that the algorithm is simple and flexible, based on swarm intelligence and has less control parameters.

ABC is expressed for finding optimal job-worker matching in JSP. According to problem definition, the problem is a variety of binary optimization problem. Due to the fact that $\mathrm{ABC}$ is basically developed for numeric problems, the algorithm is modified in such a way that it is applicable to binary space. Performance of BABC is performed on three examples of JSP known as most commonly RAP by comparing it with the BPSO. The obtained results showed that proposed approach satisfied optimum job-worker matchings.

In future work, different binary versions of $\mathrm{ABC}$ can be implemented to find optimum solutions to provide shorter computing time and can be alternatively joined effective search selection strategy to the basic algorithm definition.

\section{REFERENCES}

[1] N. Katoh and T. Ibaraki, Handbook of Combinatorial Optimization, Springer US, 1999, vol. 1-3, pp. 905-1006.

[2] S. Yamada, T. Ichimori, and M. Nishiwaki, "Optimal allocation policies for testing-resource based on a software reliability growth model,” Mathematical and Computer Modelling, vol. 22, no. (10-12), pp. 295-301, Nov. 1995.

[3] J. Turek, W. Ludwig, J. L. Wolf, L. Fleischer, P. Tiwari, J. Glasgow, U. Schwiegelsohn, and P. S. Yu, "Scheduling parallelizable tasks to minimize average response time," in SPAA '94 Proc. the Sixth Annual ACM Symposium on Parallel Algorithms and Architectures, May 1994, pp. 200-209.

[4] T.-S. Yum, M.-S. Chen, and Y.-W. Leung, "Bandwidth allocation for multimedia teleconferences," in Proc. ICC 91 International Conference on Communications Conference Record, Denver, CO, June 1991, pp. 852-858.

[5] H. Luss, "Allocation of resources of modular sizes with an application to internet protocol (IP) address allocation," Naval Research Logistics, vol. 52, no. 8, pp. 713-723, Sept. 2005.

[6] M. R. Garey, D. S. Johnson, and R. Sethi, "The complexity of flowshop and jobshop scheduling," Mathematics of Operations Research, vol. 1, no. 2, pp. 117-129, May 1976.

[7] J. Carlier and E. Pinson, "An algorithm for solving the job-shop problem,” Journal Management Science, vol. 35, no. 2, pp. 164-176, Feb. 1989.

[8] D. Applegate and W. Cook, "A computational study of the job-shop scheduling problem,” ORSA Journal on Computing, vol. 3, no. 2, pp. 149-156, Jan. 1991.

[9] P. Brucker, B. Jurisch, and B. Sievers, "A branch \& bound algorithm for the job shop scheduling problem," Discrete Applied Mathematics, vol. 49, no. 1-3, pp. 107-127, March 1991.

[10] P. J. M. V. Laarhoven, E. H. L. Aarts, and J. K. Lenstra, "Job shop scheduling by simulated annealing,” Operations Research, vol. 40, no. 1, pp. 113-125, Jan-Feb. 1992. 
[11] E. Nowicki and C. Smutnicki, "A fast taboo search algorithm for the job-shop problem,” Management Science, vol. 42, no. 6, pp. 797-813, June 1996.

[12] U. Dorndorf and E. Pesch, "Evolution based learning in a job shop environment," Computers and Operations Research, vol. 22, no. 1, pp. 25-40, Jan. 1995.

[13] G. H. Zhang, X. Y. Shao, P. G. Li, and L. Gao, “An effective hybrid particle swarm optimization algorithm for multi-objective flexible job-shop scheduling problem," Computers \& Industrial Engineering, vol. 56, no. 4, pp. 1309-1318, May 2009.

[14] K. Fan, R. Zhang, and G. Xia, "An improved particle swarm optimization algorithm and its application to a class of jsp problem," in Proc. the 2012 Fourth International Conference on Multimedia Information Networking and Security, Nov. 2012, pp. 258-261.

[15] D. Karaboga, "An idea based on honey bee swarm for numerical optimization," Technical Report TR06, Erciyes University, Engineering Faculty, Computer Engineering Department, 2005.

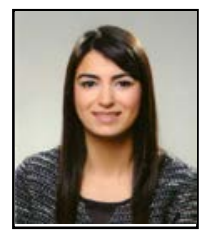

Zuleyha Yilmaz is received the B.S. degree in computer engineering from Anadolu University, Eskisehir, Turkey, in 2014. She is currently a research assistant and M.S. candidate of computer engineering in Selcuk University, Konya, Turkey. Her research interest includes optimization and optimization algorithms.

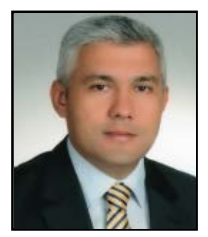

Fatih Basciftci received M.S. and Ph.D. degrees in computer systems education and electric and electronics engineering from Selcuk University, Konya, Turkey, in 2000 and 2006, respectively.

He is currently AN associate professor of computer engineering in Selcuk University, Konya, Turkey. 